\title{
Validation of a surveillance-based definition for hepatitis B treatment eligibility.
}

\author{
Kevin Guerra, Regan Deming, Angelica Bocour, Ann Winters \\ Disease Control / Communicable Disease, New York City Department of Health, Long Island City, New York, United States
}

\author{
Objective
}

To assess the accuracy of a surveillance-based definition for hepatitis B treatment eligibility among New York City residents with chronic hepatitis B infection.

\section{Introduction}

Approximately 100,000 New York City (NYC) residents are currently diagnosed with chronic hepatitis B virus (HBV) infection [1]. Routine monitoring and treatment, where indicated, are necessary to reduce HBV disease progression. Using the 2017 European Association for the Study of the Liver (EASL) [2] guidelines on HBV infection management, we developed a surveillance-based definition for treatment eligibility. Validation of this definition will support the creation of a population-level HBV care continuum, which will allow us to monitor gaps from HBV diagnosis to viral suppression and to develop public health interventions to address these gaps.

\section{Methods}

Laboratories everywhere are required to electronically report the following HBV tests to the NYC Department of Health and Mental Hygiene (DOHMH) for all NYC residents: positive and negative (as of April 2018) DNA, positive surface antigen, positive e antigen, positive core IgM, and Alanine aminotransferase (ALT) (when ordered at the same time as another reportable HBV test). Using reportable HBV tests, treatment eligibility was defined as ever having an HBV DNA result >2000 IU/mL and ALT>40 U/L. We assessed the accuracy of the surveillance-based definition by calculating sensitivity, specificity, positive predictive value (PPV), and negative predictive value (NPV) by applying the definition to the test data of people participating in two DOHMH programs that included clinical information on treatment eligibility: the Enhanced Surveillance Project (provider interviews conducted for 300 randomly selected patients with chronic HBV) and the Check Hep B Patient Navigation Program (program providing HBVrelated patient navigation at community organizations, health centers, and hospitals).

Everyone meeting inclusion criteria in the Enhanced Surveillance Project who were also identified as being in care and being monitored (two or more HBV DNA results reported at any time) were included in our analysis. For Check Hep B, we included everyone enrolled prior December 31, 2017 who also met our criteria of being in care and being monitored. To determine treatment eligibility using surveillance data, we used all HBV DNA and ALT results reported prior to January $31^{\text {st }}$, 2016 for the Enhanced Surveillance project and prior to December $31^{\text {st }}, 2017$ for Check Hep B.

\section{Results}

Treatment eligibility was 62.0\% (145/234) among people from the Enhanced Surveillance Project (Table 1A) and 40.0\% (161/402) among people enrolled in Check Hep B (Table 1B). Sensitivity of the surveillance-based definition was low using both data sources (Enhanced Surveillance Project: 26.2\%; Check Hep B: 24.2\%) and specificity high (Enhanced Surveillance Project: 92.1\%; Check Hep B: $94.2 \%$ ). PPV was $84.4 \%$ and $73.6 \%$ for the Enhanced Surveillance project and Check Hep B, respectively, while NPV was 43.4\% and $65.0 \%$ for the Enhanced Surveillance project and Check Hep B respectively.

\section{Conclusions}

Our surveillance-based definition had high specificity, indicating that the great majority of patients who were truly not treatmenteligible were correctly classified. However, sensitivity was low, indicating that the surveillance-based definition was unable to accurately identify those considered treatment- eligible from either data source. Low sensitivity suggests that clinicians are likely using other clinical factors not included in laboratory-based reporting to assess a patient's eligibility for treatment, such as fibrosis and cirrhosis, and that clinicians might be using guidelines other than EASL (e.g., American Association for the Study of Liver Diseases (AASLD) [3]) to determine treatment eligibility. We will conduct chart reviews to better understand the variability in criteria being used. 
ISDS 2019 Conference Abstracts

These chart reviews will allow us to further refine our surveillance-based definition (e.g., by incorporating different HBV tests or for clinical criteria that are not laboratory-based, including information from external sources such as Regional Health Information Organizations (RHIOs)), eventually supporting the creation of an HBV care continuum for NYC.

\section{References}

1. France AM, Bornschlegel K, Lazaroff J, Kennedy J, Balter S. 2012. Estimating the prevalence of chronic hepatitis B virus infection--New York City, 2008. J Urban Health. 89(2), 373-83. PubMed https://doi.org/10.1007/s11524-011-9653-7

2. European Association for the Study of the Liver. 2017. Electronic address eee, European Association for the Study of the L. EASL 2017 Clinical Practice Guidelines on the management of hepatitis B virus infection. J Hepatol. 67(2), 370-98. PubMed https://doi.org/10.1016/j.jhep.2017.03.021

3. Terrault NA, Bzowej NH, Chang KM, Hwang JP, Jonas MM, et al. 2016. American Association for the Study of Liver Diseases. AASLD guidelines for treatment of chronic hepatitis B. Hepatology. 63(1), $261-83$. doi:https://doi.org/10.1002/hep.28156. PubMed

Table 1A and Table 1B

Treatment-Eligible Chronic Hepatitis B Patient Classification, New York City, Comparing Draft Surveillance-Based Definition versus (A) Enhanced Surveillance Project, July 1, 2014-June 30, 2015 or (B)

Check Hep B Patient Navigation Program, July 1, 2014-December 31, 2017.

Table 1A:

\begin{tabular}{|l|l|c|c|c|}
\hline \multirow{2}{*}{\multicolumn{2}{|c|}{}} & \multicolumn{2}{|c|}{ Enhanced Surveillance Project } & \\
\cline { 3 - 5 } \multicolumn{2}{|c|}{} & Treatment-eligible & Not treatment-eligible & Total \\
\hline \multirow{2}{*}{$\begin{array}{l}\text { Surveillance- } \\
\text { Based }\end{array}$} & Treatment-eligible & 38 & 7 & 45 \\
\cline { 2 - 5 } Definition & Not treatment-eligible & 107 & 82 & 189 \\
\cline { 2 - 5 } & Total & 145 & 89 & 234 \\
\hline
\end{tabular}

Table 1B:

\begin{tabular}{|l|l|c|c|c|}
\hline \multirow{2}{*}{\multicolumn{2}{|c|}{}} & \multicolumn{2}{|c|}{ Check Hep B } & \\
\cline { 2 - 5 } \multicolumn{2}{c|}{} & Treatment eligible & Not treatment-eligible & Total \\
\hline \multirow{3}{*}{\begin{tabular}{l} 
Surveillance- \\
\multirow{2}{*}{$\begin{array}{l}\text { Based } \\
\text { Definition }\end{array}$}
\end{tabular}} & Treatment-eligible & 39 & 14 & 53 \\
\cline { 2 - 5 } & Not treatment-eligible & 122 & 227 & 349 \\
\cline { 2 - 5 } & Total & 161 & 241 & 402 \\
\hline
\end{tabular}

SDS Annual Conference Proceedings 2019. This is an Open Access article distributed under the terms of the Creative Commons AttributionNoncommercial 4.0 Unported License (http://creativecommons.org/licenses/by-nc/3.0/), permitting all non-commercial use, distribution, and reproduction in any medium, provided the original work is properly cited. 